\title{
Observation of $B^{+} \rightarrow a_{1}^{+}(1260) K^{0}$ and $B^{0} \rightarrow a_{1}^{-}(1260) K^{+}$
}

B. Aubert, ${ }^{1}$ M. Bona, ${ }^{1}$ D. Boutigny, ${ }^{1}$ Y. Karyotakis, ${ }^{1}$ J. P. Lees,${ }^{1}$ V. Poireau, ${ }^{1}$ X. Prudent, ${ }^{1}$ V. Tisserand, ${ }^{1}$ A. Zghiche, ${ }^{1}$ J. Garra Tico, ${ }^{2}$ E. Grauges, ${ }^{2}$ L. Lopez,${ }^{3}$ A. Palano, ${ }^{3}$ M. Pappagallo, ${ }^{3}$ G. Eigen, ${ }^{4}$ B. Stugu, ${ }^{4}$ L. Sun,${ }^{4}$ G. S. Abrams, ${ }^{5}$ M. Battaglia, ${ }^{5}$ D. N. Brown, ${ }^{5}$ J. Button-Shafer, ${ }^{5}$ R. N. Cahn, ${ }^{5}$ Y. Groysman, ${ }^{5}$ R. G. Jacobsen, ${ }^{5}$ J. A. Kadyk, ${ }^{5}$ L. T. Kerth, ${ }^{5}$ Yu. G. Kolomensky, ${ }^{5}$ G. Kukartsev, ${ }^{5}$ D. Lopes Pegna, ${ }^{5}$ G. Lynch, ${ }^{5}$ L. M. Mir, ${ }^{5}$ T. J. Orimoto, ${ }^{5}$ I. L. Osipenkov, ${ }^{5}$

M. T. Ronan, ${ }^{5, *}$ K. Tackmann, ${ }^{5}$ T. Tanabe, ${ }^{5}$ W. A. Wenzel, ${ }^{5}$ P. del Amo Sanchez,${ }^{6}$ C. M. Hawkes, ${ }^{6}$ A. T. Watson, ${ }^{6}$ H. Koch,

T. Schroeder, ${ }^{7}$ D. Walker ${ }^{8}$ D. J. Asgeirsson, ${ }^{9}$ T. Cuhadar-Donszelmann, ${ }^{9}$ B. G. Fulsom, ${ }^{9}$ C. Hearty, ${ }^{9}$ T. S. Mattison, ${ }^{9}$ J. A. McKenna, ${ }^{9}$ M. Barrett, ${ }^{10}$ A. Khan,${ }^{10}$ M. Saleem,${ }^{10}$ L. Teodorescu, ${ }^{10}$ V. E. Blinov,${ }^{11}$ A. D. Bukin, ${ }^{11}$ V. P. Druzhinin, ${ }^{11}$ V. B. Golubev, ${ }^{11}$ A. P. Onuchin, ${ }^{11}$ S. I. Serednyakov, ${ }^{11}$ Yu. I. Skovpen, ${ }^{11}$ E. P. Solodov,${ }^{11}$ K. Yu. Todyshev,${ }^{11}$ M. Bondioli, ${ }^{12}$ S. Curry, ${ }^{12}$ I. Eschrich, ${ }^{12}$ D. Kirkby, ${ }^{12}$ A. J. Lankford, ${ }^{12}$ P. Lund, ${ }^{12}$ M. Mandelkern, ${ }^{12}$ E. C. Martin, ${ }^{12}$ D. P. Stoker, ${ }^{12}$ S. Abachi, ${ }^{13}$ C. Buchanan, ${ }^{13}$ S. D. Foulkes, ${ }^{14}$ J. W. Gary, ${ }^{14}$ F. Liu,,${ }^{14}$ O. Long, ${ }^{14}$ B. C. Shen,${ }^{14}$ G. M. Vitug, ${ }^{14}$ L. Zhang,${ }^{14}$ H. P. Paar, ${ }^{15}$ S. Rahatlou, ${ }^{15}$ V. Sharma,${ }^{15}$ J. W. Berryhill, ${ }^{16}$ C. Campagnari, ${ }^{16}$ A. Cunha, ${ }^{16}$ B. Dahmes, ${ }^{16}$ T. M. Hong, ${ }^{16}$

D. Kovalskyi, ${ }^{16}$ J. D. Richman, ${ }^{16}$ T. W. Beck,${ }^{17}$ A. M. Eisner, ${ }^{17}$ C. J. Flacco,,${ }^{17}$ C. A. Heusch,,${ }^{17}$ J. Kroseberg, ${ }^{17}$ W. S. Lockman, ${ }^{17}$ T. Schalk, ${ }^{17}$ B. A. Schumm, ${ }^{17}$ A. Seiden, ${ }^{17}$ M. G. Wilson, ${ }^{17}$ L. O. Winstrom,,${ }^{17}$ E. Chen, ${ }^{18}$ C. H. Cheng, ${ }^{18}$ F. Fang, ${ }^{18}$ D. G. Hitlin, ${ }^{18}$ I. Narsky, ${ }^{18}$ T. Piatenko, ${ }^{18}$ F. C. Porter, ${ }^{18}$ R. Andreassen, ${ }^{19}$ G. Mancinelli, ${ }^{19}$ B. T. Meadows,${ }^{19}$ K. Mishra, ${ }^{19}$ M. D. Sokoloff, ${ }^{19}$ F. Blanc,${ }^{20}$ P. C. Bloom, ${ }^{20}$ S. Chen, ${ }^{20}$ W. T. Ford,${ }^{20}$ J. F. Hirschauer,${ }^{20}$ A. Kreisel, ${ }^{20}$ M. Nagel, ${ }^{20}$ U. Nauenberg, ${ }^{20}$ A. Olivas, ${ }^{20}$ J. G. Smith, ${ }^{20}$ K. A. Ulmer, ${ }^{20}$ S. R. Wagner, ${ }^{20}$ J. Zhang, ${ }^{20}$ A. M. Gabareen, ${ }^{21}$ A. Soffer, ${ }^{21, \dagger}$ W. H. Toki, ${ }^{21}$ R. J. Wilson, ${ }^{21}$ F. Winklmeier, ${ }^{21}$ D. D. Altenburg, ${ }^{22}$ E. Feltresi, ${ }^{22}$ A. Hauke, ${ }^{22}$ H. Jasper, ${ }^{22}$ J. Merkel, ${ }^{22}$ A. Petzold, ${ }^{22}$ B. Spaan, ${ }^{22}$ K. Wacker, ${ }^{22}$ V. Klose, ${ }^{23}$ M. J. Kobel, ${ }^{23}$ H. M. Lacker, ${ }^{23}$ W. F. Mader, ${ }^{23}$

R. Nogowski, ${ }^{23}$ J. Schubert, ${ }^{23}$ K. R. Schubert, ${ }^{23}$ R. Schwierz, ${ }^{23}$ J. E. Sundermann, ${ }^{23}$ A. Volk, ${ }^{23}$ D. Bernard, ${ }^{24}$ G. R. Bonneaud, ${ }^{24}$ E. Latour, ${ }^{24}$ V. Lombardo, ${ }^{24}$ Ch. Thiebaux,${ }^{24}$ M. Verderi, ${ }^{24}$ P. J. Clark, ${ }^{25}$ W. Gradl,,${ }^{25}$ F. Muheim, ${ }^{25}$ S. Playfer, ${ }^{25}$ A. I. Robertson, ${ }^{25}$ J. E. Watson, ${ }^{25}$ Y. Xie, ${ }^{25}$ M. Andreotti ${ }^{26}$ D. Bettoni, ${ }^{26}$ C. Bozzi,${ }^{26}$ R. Calabrese, ${ }^{26}$ A. Cecchi ${ }^{26}$ G. Cibinetto, ${ }^{26}$ P. Franchini, ${ }^{26}$ E. Luppi ${ }^{26}$ M. Negrini,${ }^{26}$ A. Petrella, ${ }^{26}$ L. Piemontese,${ }^{26}$ E. Prencipe ${ }^{26}$ V. Santoro, ${ }^{26}$ F. Anulli, ${ }^{27}$ R. Baldini-Ferroli, ${ }^{27}$ A. Calcaterra, ${ }^{27}$ R. de Sangro, ${ }^{27}$ G. Finocchiaro, ${ }^{27}$ S. Pacetti, ${ }^{27}$ P. Patteri, ${ }^{27}$ I. M. Peruzzi, ${ }^{27, \$}$ M. Piccolo, ${ }^{27}$ M. Rama,${ }^{27}$ A. Zallo, ${ }^{27}$ A. Buzzo, ${ }^{28}$ R. Contri,${ }^{28}$ M. Lo Vetere, ${ }^{28}$ M. M. Macri, ${ }^{28}$ M. R. Monge, ${ }^{28}$ S. Passaggio, ${ }^{28}$ C. Patrignani, ${ }^{28}$ E. Robutti, ${ }^{28}$ A. Santroni, ${ }^{28}$ S. Tosi, ${ }^{28}$ K. S. Chaisanguanthum, ${ }^{29}$ M. Morii, ${ }^{29}$ J. Wu, ${ }^{29}$ R. S. Dubitzky, ${ }^{30}$ J. Marks, ${ }^{30}$ S. Schenk ${ }^{30}$ U. Uwer, ${ }^{30}$ D. J. Bard, ${ }^{31}$ P. D. Dauncey,${ }^{31}$ R. L. Flack, ${ }^{31}$ J. A. Nash, ${ }^{31}$ W. Panduro Vazquez, ${ }^{31}$ M. Tibbetts, ${ }^{31}$ P. K. Behera, ${ }^{32}$ X. Chai, ${ }^{32}$ M. J. Charles, ${ }^{32}$ U. Mallik, ${ }^{32}$ J. Cochran, ${ }^{33}$ H. B. Crawley, ${ }^{33}$ L. Dong, ${ }^{33}$ V. Eyges, ${ }^{33}$ W. T. Meyer, ${ }^{33}$ S. Prell, ${ }^{33}$ E. I. Rosenberg, ${ }^{33}$ A. E. Rubin, ${ }^{33}$ Y. Y. Gao, ${ }^{34}$ A. V. Gritsan, ${ }^{34}$ Z. J. Guo ${ }^{34}$ C. K. Lae, ${ }^{34}$ A. G. Denig, ${ }^{35}$ M. Fritsch, ${ }^{35}$ G. Schott, ${ }^{35}$ N. Arnaud,${ }^{36}$ J. Béquilleux, ${ }^{36}$ A. D’Orazio, ${ }^{36}$ M. Davier, ${ }^{36}$ G. Grosdidier, ${ }^{36}$ A. Höcker, ${ }^{36}$ V. Lepeltier, ${ }^{36}$ F. Le Diberder, ${ }^{36}$ A. M. Lutz, ${ }^{36}$ S. Pruvot, ${ }^{36}$ S. Rodier, ${ }^{36}$ P. Roudeau, ${ }^{36}$ M. H. Schune, ${ }^{36}$ J. Serrano, ${ }^{36}$ V. Sordini, ${ }^{36}$ A. Stocchi, ${ }^{36}$ W. F. Wang, ${ }^{36}$ G. Wormser, ${ }^{36}$ D. J. Lange, ${ }^{37}$ D. M. Wright, ${ }^{37}$ I. Bingham, ${ }^{38}$ J. P. Burke, ${ }^{38}$ C. A. Chavez, ${ }^{38}$ J. R. Fry, ${ }^{38}$ E. Gabathuler, ${ }^{38}$ R. Gamet, ${ }^{38}$ D. E. Hutchcroft, ${ }^{38}$ D. J. Payne, ${ }^{38}$ K. C. Schofield, ${ }^{38}$ C. Touramanis, ${ }^{38}$ A. J. Bevan, ${ }^{39}$ K. A. George,${ }^{39}$ F. Di Lodovico, ${ }^{39}$

R. Sacco, ${ }^{39}$ G. Cowan,${ }^{40}$ H. U. Flaecher, ${ }^{40}$ D. A. Hopkins,${ }^{40}$ S. Paramesvaran,${ }^{40}$ F. Salvatore, ${ }^{40}$ A. C. Wren, ${ }^{40}$

D. N. Brown, ${ }^{41}$ C. L. Davis, ${ }^{41}$ J. Allison, ${ }^{42}$ D. Bailey, ${ }^{42}$ N. R. Barlow, ${ }^{42}$ R. J. Barlow, ${ }^{42}$ Y. M. Chia, ${ }^{42}$ C. L. Edgar, ${ }^{42}$ G. D. Lafferty, ${ }^{42}$ T. J. West, ${ }^{42}$ J. I. Yi ${ }^{42}$ J. Anderson, ${ }^{43}$ C. Chen, ${ }^{43}$ A. Jawahery, ${ }^{43}$ D. A. Roberts, ${ }^{43}$ G. Simi,${ }^{43}$ J. M. Tuggle, ${ }^{43}$ G. Blaylock, ${ }^{44}$ C. Dallapiccola, ${ }^{44}$ S. S. Hertzbach,${ }^{44}$ X. Li, ${ }^{44}$ T. B. Moore,,${ }^{44}$ E. Salvati, ${ }^{44}$ S. Saremi, ${ }^{44}$ R. Cowan, ${ }^{45}$ D. Dujmic, ${ }^{45}$ P. H. Fisher ${ }^{45}$ K. Koeneke, ${ }^{45}$ G. Sciolla, ${ }^{45}$ M. Spitznagel,${ }^{45}$ F. Taylor, ${ }^{45}$ R. K. Yamamoto ${ }^{45}$ M. Zhao, ${ }^{45}$ Y. Zheng, ${ }^{45}$ S. E. Mclachlin, ${ }^{46, *}$ P. M. Patel,${ }^{46}$ S. H. Robertson, ${ }^{46}$ A. Lazzaro, ${ }^{47}$ F. Palombo, ${ }^{47}$ J. M. Bauer, ${ }^{48}$ L. Cremaldi, ${ }^{48}$ V. Eschenburg, ${ }^{48}$ R. Godang, ${ }^{48}$ R. Kroeger, ${ }^{48}$ D. A. Sanders, ${ }^{48}$ D. J. Summers, ${ }^{48}$ H. W. Zhao, ${ }^{48}$ S. Brunet, ${ }^{49}$ D. Côté ${ }^{49}$ M. Simard ${ }^{49}$ P. Taras, ${ }^{49}$ F. B. Viaud, ${ }^{49}$ H. Nicholson, ${ }^{50}$ G. De Nardo, ${ }^{51}$ F. Fabozzi, ${ }^{51,}$ L. Lista, ${ }^{51}$ D. Monorchio, ${ }^{51}$ C. Sciacca, ${ }^{51}$ M. A. Baak, ${ }^{52}$ G. Raven, ${ }^{52}$ H. L. Snoek, ${ }^{52}$ C. P. Jessop ${ }^{53}$ K. J. Knoepfel, ${ }^{53}$ J. M. LoSecco, ${ }^{53}$ G. Benelli, ${ }^{54}$ L. A. Corwin, ${ }^{54}$ K. Honscheid ${ }^{54}$ H. Kagan,${ }^{54}$ R. Kass, ${ }^{54}$ J. P. Morris, ${ }^{54}$ A. M. Rahimi,${ }^{54}$ J. J. Regensburger,${ }^{54}$ S. J. Sekula,${ }^{54}$ Q. K. Wong, ${ }^{54}$ N. L. Blount, ${ }^{55}$ J. Brau, ${ }^{55}$ R. Frey, ${ }^{55}$ O. Igonkina, ${ }^{55}$ J. A. Kolb,${ }^{55}$ M. Lu, ${ }^{55}$ R. Rahmat, ${ }^{55}$ N. B. Sinev, ${ }^{55}$

D. Strom, ${ }^{55}$ J. Strube,${ }^{55}$ E. Torrence, ${ }^{55}$ N. Gagliardi,${ }^{56}$ A. Gaz ${ }^{56}$ M. Margoni, ${ }^{56}$ M. Morandin, ${ }^{56}$ A. Pompili, ${ }^{56}$

M. Posocco, ${ }^{56}$ M. Rotondo, ${ }^{56}$ F. Simonetto,${ }^{56}$ R. Stroili, ${ }^{56}$ C. Voci, ${ }^{56}$ E. Ben-Haim, ${ }^{57}$ H. Briand ${ }^{57}$ G. Calderini,${ }^{57}$

J. Chauveau, ${ }^{57}$ P. David ${ }^{57}$ L. Del Buono,${ }^{57}$ Ch. de la Vaissière, ${ }^{57}$ O. Hamon, ${ }^{57}$ Ph. Leruste,${ }^{57}$ J. Malclès,${ }^{57}$ J. Ocariz, ${ }^{57}$ A. Perez, ${ }^{57}$ J. Prendki, ${ }^{57}$ L. Gladney, ${ }^{58}$ M. Biasini, ${ }^{59}$ R. Covarelli, ${ }^{59}$ E. Manoni,${ }^{59}$ C. Angelini, ${ }^{60}$ G. Batignani, ${ }^{60}$ 
S. Bettarini, ${ }^{60}$ M. Carpinelli, ${ }^{60}$ R. Cenci,${ }^{60}$ A. Cervelli, ${ }^{60}$ F. Forti ${ }^{60}$ M. A. Giorgi,${ }^{60}$ A. Lusiani, ${ }^{60}$ G. Marchiori, ${ }^{60}$ M. A. Mazur, ${ }^{60}$ M. Morganti, ${ }^{60}$ N. Neri,${ }^{60}$ E. Paoloni, ${ }^{60}$ G. Rizzo, ${ }^{60}$ J. J. Walsh, ${ }^{60}$ J. Biesiada, ${ }^{61}$ P. Elmer, ${ }^{61}$ Y.P. Lau, ${ }^{61}$ C. Lu, ${ }^{61}$ J. Olsen, ${ }^{61}$ A. J. S. Smith, ${ }^{61}$ A. V. Telnov, ${ }^{61}$ E. Baracchini, ${ }^{62}$ F. Bellini, ${ }^{62}$ G. Cavoto, ${ }^{62}$ D. del Re,${ }^{62}$ E. Di Marco,${ }^{62}$ R. Faccini, ${ }^{62}$ F. Ferrarotto, ${ }^{62}$ F. Ferroni, ${ }^{62}$ M. Gaspero, ${ }^{62}$ P. D. Jackson, ${ }^{62}$ L. Li Gioi ${ }^{62}$ M. A. Mazzoni, ${ }^{62}$ S. Morganti, ${ }^{62}$ G. Piredda, ${ }^{62}$ F. Polci, ${ }^{62}$ F. Renga,${ }^{62}$ C. Voena,${ }^{62}$ M. Ebert, ${ }^{63}$ T. Hartmann, ${ }^{63}$ H. Schröder,${ }^{63}$ R. Waldi, ${ }^{63}$ T. Adye,${ }^{64}$ G. Castelli, ${ }^{64}$ B. Franek, ${ }^{64}$ E. O. Olaiya,${ }^{64}$ W. Roethel, ${ }^{64}$ F. F. Wilson, ${ }^{64}$ S. Emery, ${ }^{65}$ M. Escalier,${ }^{65}$ A. Gaidot,${ }^{65}$ S. F. Ganzhur, ${ }^{65}$ G. Hamel de Monchenault, ${ }^{65}$ W. Kozanecki, ${ }^{65}$ G. Vasseur,${ }^{65}$ Ch. Yèche,${ }^{65}$ M. Zito, ${ }^{65}$ X. R. Chen, ${ }^{66}$ H. Liu, ${ }^{66}$ W. Park, ${ }^{66}$ M. V. Purohit, ${ }^{66}$ R. M. White, ${ }^{66}$ J. R. Wilson, ${ }^{66}$ M. T. Allen, ${ }^{67}$ D. Aston, ${ }^{67}$ R. Bartoldus,${ }^{67}$ P. Bechtle,${ }^{67}$ R. Claus, ${ }^{67}$ J. P. Coleman, ${ }^{67}$ M. R. Convery, ${ }^{67}$ J. C. Dingfelder ${ }^{67}$ J. Dorfan, ${ }^{67}$ G. P. Dubois-Felsmann, ${ }^{67}$ W. Dunwoodie ${ }^{67}$ R. C. Field, ${ }^{67}$ T. Glanzman, ${ }^{67}$ S. J. Gowdy, ${ }^{67}$ M. T. Graham, ${ }^{67}$ P. Grenier, ${ }^{67}$ C. Hast, ${ }^{67}$ W. R. Innes, ${ }^{67}$ J. Kaminski, ${ }^{67}$ M. H. Kelsey, ${ }^{67}$ H. Kim, ${ }^{67}$ P. Kim,${ }^{67}$ M. L. Kocian,${ }^{67}$ D. W. G. S. Leith, ${ }^{67}$ S. Li, ${ }^{67}$ S. Luitz, ${ }^{67}$ V. Luth, ${ }^{67}$ H. L. Lynch, ${ }^{67}$ D. B. MacFarlane, ${ }^{67}$ H. Marsiske, ${ }^{67}$ R. Messner, ${ }^{67}$ D. R. Muller, ${ }^{67}$ C. P. O'Grady ${ }^{67}$ I. Ofte, ${ }^{67}$ A. Perazzo, ${ }^{67}$ M. Perl, ${ }^{67}$ T. Pulliam, ${ }^{67}$ B. N. Ratcliff, ${ }^{67}$ A. Roodman, ${ }^{67}$ A. A. Salnikov, ${ }^{67}$ R. H. Schindler, ${ }^{67}$ J. Schwiening, ${ }^{67}$ A. Snyder, ${ }^{67}$ D. Su, ${ }^{67}$ M. K. Sullivan, ${ }^{67}$ K. Suzuki, ${ }^{67}$ S. K. Swain,${ }^{67}$ J. M. Thompson, ${ }^{67}$ J. Va'vra, ${ }^{67}$ A. P. Wagner, ${ }^{67}$ M. Weaver, ${ }^{67}$ W. J. Wisniewski, ${ }^{67}$ M. Wittgen ${ }^{67}$ D. H. Wright, ${ }^{67}$ A. K. Yarritu, ${ }^{67}$ K. Yi, ${ }^{67}$ C. C. Young, ${ }^{67}$ V. Ziegler, ${ }^{67}$ P. R. Burchat,${ }^{68}$ A. J. Edwards, ${ }^{68}$ S. A. Majewski, ${ }^{68}$ T. S. Miyashita, ${ }^{68}$ B. A. Petersen, ${ }^{68}$ L. Wilden, ${ }^{68}$ S. Ahmed, ${ }^{69}$ M. S. Alam, ${ }^{69}$ R. Bula, ${ }^{69}$ J. A. Ernst, ${ }^{69}$ V. Jain, ${ }^{69}$ B. Pan, ${ }^{69}$ M. A. Saeed, ${ }^{69}$ F. R. Wappler, ${ }^{69}$ S. B. Zain, ${ }^{69}$ M. Krishnamurthy, ${ }^{70}$ S. M. Spanier,${ }^{70}$ R. Eckmann, ${ }^{71}$ J. L. Ritchie, ${ }^{71}$ A. M. Ruland, ${ }^{71}$ C. J. Schilling, ${ }^{71}$ R. F. Schwitters,${ }^{71}$ J. M. Izen, ${ }^{72}$ X. C. Lou, ${ }^{72}$ S. Ye, ${ }^{72}$ F. Bianchi, ${ }^{73}$ F. Gallo, ${ }^{73}$ D. Gamba,${ }^{73}$ M. Pelliccioni,${ }^{73}$ M. Bomben,${ }^{74}$ L. Bosisio,${ }^{74}$ C. Cartaro, ${ }^{74}$ F. Cossutti,${ }^{74}$ G. Della Ricca, ${ }^{74}$ L. Lanceri, ${ }^{74}$ L. Vitale,${ }^{74}$ V. Azzolini,${ }^{75}$ N. Lopez-March, ${ }^{75}$ F. Martinez-Vidal,${ }^{75, \mid}$ D. A. Milanes, ${ }^{75}$ A. Oyanguren, ${ }^{75}$ J. Albert, ${ }^{76}$ Sw. Banerjee,${ }^{76}$ B. Bhuyan, ${ }^{76}$ K. Hamano,${ }^{76}$ R. Kowalewski, ${ }^{76}$ I. M. Nugent, ${ }^{76}$ J. M. Roney, ${ }^{76}$ R. J. Sobie ${ }^{76}$ P. F. Harrison, ${ }^{77}$ J. Ilic, ${ }^{77}$ T. E. Latham,${ }^{77}$ G. B. Mohanty, ${ }^{77}$ H. R. Band,${ }^{78}$ X. Chen,${ }^{78}$ S. Dasu,${ }^{78}$ K. T. Flood, ${ }^{78}$ J. J. Hollar, ${ }^{78}$ P. E. Kutter, ${ }^{78}$ Y. Pan,${ }^{78}$ M. Pierini, ${ }^{78}$ R. Prepost, ${ }^{78}$ S. L. Wu, ${ }^{78}$ and H. Neal ${ }^{79}$

(BABAR Collaboration)

\footnotetext{
${ }^{1}$ Laboratoire de Physique des Particules, IN2P3/CNRS et Université de Savoie, F-74941 Annecy-Le-Vieux, France

${ }^{2}$ Universitat de Barcelona, Facultat de Fisica, Departament ECM, E-08028 Barcelona, Spain

${ }^{3}$ Università di Bari, Dipartimento di Fisica and INFN, I-70126 Bari, Italy

${ }^{4}$ University of Bergen, Institute of Physics, N-5007 Bergen, Norway

${ }^{5}$ Lawrence Berkeley National Laboratory and University of California, Berkeley, California 94720, USA

${ }^{6}$ University of Birmingham, Birmingham, B15 2TT, United Kingdom

${ }^{7}$ Ruhr Universität Bochum, Institut für Experimentalphysik 1, D-44780 Bochum, Germany

${ }^{8}$ University of Bristol, Bristol BS8 1TL, United Kingdom

${ }^{9}$ University of British Columbia, Vancouver, British Columbia, Canada V6T IZ1

${ }^{10}$ Brunel University, Uxbridge, Middlesex UB8 3PH, United Kingdom

${ }^{11}$ Budker Institute of Nuclear Physics, Novosibirsk 630090, Russia

${ }^{12}$ University of California at Irvine, Irvine, California 92697, USA

${ }^{13}$ University of California at Los Angeles, Los Angeles, California 90024, USA

${ }^{14}$ University of California at Riverside, Riverside, California 92521, USA

${ }^{15}$ University of California at San Diego, La Jolla, California 92093, USA

${ }^{16}$ University of California at Santa Barbara, Santa Barbara, California 93106, USA

${ }^{17}$ University of California at Santa Cruz, Institute for Particle Physics, Santa Cruz, California 95064, USA

${ }^{18}$ California Institute of Technology, Pasadena, California 91125, USA

${ }^{19}$ University of Cincinnati, Cincinnati, Ohio 45221, USA

${ }^{20}$ University of Colorado, Boulder, Colorado 80309, USA

${ }^{21}$ Colorado State University, Fort Collins, Colorado 80523, USA

${ }^{22}$ Universität Dortmund, Institut für Physik, D-44221 Dortmund, Germany

${ }^{23}$ Technische Universität Dresden, Institut für Kern- und Teilchenphysik, D-01062 Dresden, Germany

${ }^{24}$ Laboratoire Leprince-Ringuet, CNRS/IN2P3, Ecole Polytechnique, F-91128 Palaiseau, France

${ }^{25}$ University of Edinburgh, Edinburgh EH9 3JZ, United Kingdom

${ }^{26}$ Università di Ferrara, Dipartimento di Fisica and INFN, I-44100 Ferrara, Italy

${ }^{27}$ Laboratori Nazionali di Frascati dell'INFN, I-00044 Frascati, Italy

${ }^{28}$ Università di Genova, Dipartimento di Fisica and INFN, I-16146 Genova, Italy

${ }^{29}$ Harvard University, Cambridge, Massachusetts 02138, USA

${ }^{30}$ Universität Heidelberg, Physikalisches Institut, Philosophenweg 12, D-69120 Heidelberg, Germany
} 
${ }^{31}$ Imperial College London, London, SW7 2AZ, United Kingdom

${ }^{32}$ University of Iowa, Iowa City, Iowa 52242, USA

${ }^{33}$ Iowa State University, Ames, Iowa 50011-3160, USA

${ }^{34}$ Johns Hopkins University, Baltimore, Maryland 21218, USA

${ }^{35}$ Universität Karlsruhe, Institut für Experimentelle Kernphysik, D-76021 Karlsruhe, Germany

${ }^{36}$ Laboratoire de l'Accélérateur Linéaire, IN2P3/CNRS et Université Paris-Sud 11, Centre Scientifique d'Orsay, B.P. 34, F-91898 ORSAY Cedex, France

${ }^{37}$ Lawrence Livermore National Laboratory, Livermore, California 94550, USA

${ }^{38}$ University of Liverpool, Liverpool L69 7ZE, United Kingdom

${ }^{39}$ Queen Mary, University of London, E1 4NS, United Kingdom

${ }^{40}$ University of London, Royal Holloway and Bedford New College, Egham, Surrey TW20 OEX, United Kingdom

${ }^{41}$ University of Louisville, Louisville, Kentucky 40292, USA

${ }^{42}$ University of Manchester, Manchester M13 9PL, United Kingdom

${ }^{43}$ University of Maryland, College Park, Maryland 20742, USA

${ }^{44}$ University of Massachusetts, Amherst, Massachusetts 01003, USA

${ }^{45}$ Massachusetts Institute of Technology, Laboratory for Nuclear Science, Cambridge, Massachusetts 02139, USA

${ }^{46}$ McGill University, Montréal, Québec, Canada H3A $2 T 8$

${ }^{47}$ Università di Milano, Dipartimento di Fisica and INFN, I-20133 Milano, Italy

${ }^{48}$ University of Mississippi, University, Mississippi 38677, USA

${ }^{49}$ Université de Montréal, Physique des Particules, Montréal, Québec, Canada H3C 3J7

${ }^{50}$ Mount Holyoke College, South Hadley, Massachusetts 01075, USA

${ }^{51}$ Università di Napoli Federico II, Dipartimento di Scienze Fisiche and INFN, I-80126, Napoli, Italy

${ }^{52}$ NIKHEF, National Institute for Nuclear Physics and High Energy Physics, NL-1009 DB Amsterdam, The Netherlands

${ }^{53}$ University of Notre Dame, Notre Dame, Indiana 46556, USA

${ }^{54}$ The Ohio State University, Columbus, Ohio 43210, USA

${ }^{55}$ University of Oregon, Eugene, Oregon 97403, USA

${ }^{56}$ Università di Padova, Dipartimento di Fisica and INFN, I-35131 Padova, Italy

${ }^{57}$ Laboratoire de Physique Nucléaire et de Hautes Energies, IN2P3/CNRS, Université Pierre et Marie Curie-Paris6, Université Denis Diderot-Paris7, F-75252 Paris, France

${ }^{58}$ University of Pennsylvania, Philadelphia, Pennsylvania 19104, USA

${ }^{59}$ Università di Perugia, Dipartimento di Fisica and INFN, I-06100 Perugia, Italy

${ }^{60}$ Università di Pisa, Dipartimento di Fisica, Scuola Normale Superiore and INFN, I-56127 Pisa, Italy

${ }^{61}$ Princeton University, Princeton, New Jersey 08544, USA

${ }^{62}$ Università di Roma La Sapienza, Dipartimento di Fisica and INFN, I-00185 Roma, Italy

${ }^{63}$ Universität Rostock, D-18051 Rostock, Germany

${ }^{64}$ Rutherford Appleton Laboratory, Chilton, Didcot, Oxon, OX11 OQX, United Kingdom

${ }^{65}$ DSM/Dapnia, CEA/Saclay, F-91191 Gif-sur-Yvette, France

${ }^{66}$ University of South Carolina, Columbia, South Carolina 29208, USA

${ }^{67}$ Stanford Linear Accelerator Center, Stanford, California 94309, USA

${ }^{68}$ Stanford University, Stanford, California 94305-4060, USA

${ }^{69}$ State University of New York, Albany, New York 12222, USA

${ }^{70}$ University of Tennessee, Knoxville, Tennessee 37996, USA

${ }^{71}$ University of Texas at Austin, Austin, Texas 78712, USA

${ }^{72}$ University of Texas at Dallas, Richardson, Texas 75083, USA

${ }^{73}$ Università di Torino, Dipartimento di Fisica Sperimentale and INFN, I-10125 Torino, Italy

${ }^{74}$ Università di Trieste, Dipartimento di Fisica and INFN, I-34127 Trieste, Italy

${ }^{75}$ IFIC, Universitat de Valencia-CSIC, E-46071 Valencia, Spain

${ }^{76}$ University of Victoria, Victoria, British Columbia, Canada V8W $3 P 6$

${ }^{77}$ Department of Physics, University of Warwick, Coventry CV4 7AL, United Kingdom

${ }^{78}$ University of Wisconsin, Madison, Wisconsin 53706, USA

${ }^{79}$ Yale University, New Haven, Connecticut 06511, USA

(Received 27 September 2007; published 8 February 2008)

We present branching fraction measurements of the decays $B^{+} \rightarrow a_{1}^{+}(1260) K^{0}$ and $B^{0} \rightarrow a_{1}^{-}(1260) K^{+}$ with $a_{1}^{ \pm}(1260) \rightarrow \pi^{\mp} \pi^{ \pm} \pi^{ \pm}$. The data sample corresponds to $383 \times 10^{6} B \bar{B}$ pairs produced in $e^{+} e^{-}$ annihilation through the $\Upsilon(4 S)$ resonance. We measure the products of the branching fractions $\mathcal{B}\left(B^{+} \rightarrow a_{1}^{+}(1260) K^{0}\right) \mathcal{B}\left(a_{1}^{+}(1260) \rightarrow \pi^{-} \pi^{+} \pi^{+}\right)=(17.4 \pm 2.5 \pm 2.2) \times 10^{-6} \quad$ and $\quad \mathcal{B}\left(B^{0} \rightarrow\right.$ $\left.a_{1}^{-}(1260) K^{+}\right) \mathcal{B}\left(a_{1}^{-}(1260) \rightarrow \pi^{+} \pi^{-} \pi^{-}\right)=(8.2 \pm 1.5 \pm 1.2) \times 10^{-6}$. We also measure the charge asymmetries $\mathcal{A}_{\mathrm{ch}}\left(B^{+} \rightarrow a_{1}^{+}(1260) K^{0}\right)=0.12 \pm 0.11 \pm 0.02$ and $\mathcal{A}_{\mathrm{ch}}\left(B^{0} \rightarrow a_{1}^{-}(1260) K^{+}\right)=-0.16 \pm$ $0.12 \pm 0.01$. The first uncertainty quoted is statistical and the second is systematic. 
Recently the BABAR Collaboration has reported the measurement of the branching fraction [1] and timedependent $C P$-violation parameters for the process $B^{0} \rightarrow$ $a_{1}^{ \pm}(1260) \pi^{\mp}$ [2]. If this process were mediated by a single tree amplitude these measured parameters would enable a determination of the angle $\alpha$ of the unitary triangle of the Cabibbo-Kobayashi-Maskawa (CKM) quark-mixing matrix [3]. However, in the presence of a penguin amplitude with a different weak phase from the tree amplitude the measured angle, called $\alpha_{\text {eff }}$, would differ from $\alpha$ [4]. An upper bound on the difference $\Delta \alpha=\left|\alpha-\alpha_{\text {eff }}\right|$ can be calculated using SU(3) together with measurements of the $C P$-averaged decay rates for either the decays $B^{+} \rightarrow$ $a_{1}^{+}(1260) K^{0}$ or $B^{0} \rightarrow a_{1}^{-}(1260) K^{+}$and the decays $B \rightarrow$ $K_{1}(1270) \pi$ and $B \rightarrow K_{1}(1400) \pi$ [5]. Knowing the value of this difference is important in calculating bounds on the angle $\alpha$.

There are no experimental measurements of the branching fractions of the decays $B^{+} \rightarrow a_{1}^{+}(1260) K^{0}$ and $B^{0} \rightarrow$ $a_{1}^{-}(1260) K^{+}$to this date. Recent theoretical estimates of these branching fractions have been calculated assuming QCD factorization [6] as well as naive factorization for two different values of the mixing angle $\theta$ between the two strange $P$ wave axial mesons [7]. The estimated branching fractions lie in the range $(16-52) \times 10^{-6}[8]$. Comparison between theoretical predictions and measured quantities is useful to test the underlying theoretical hypotheses of factorization and $B \rightarrow a_{1}(1260)$ transition form factors.

We present measurements of the branching fraction for the decays $B^{+} \rightarrow a_{1}^{+}(1260) K^{0}$ and $B^{0} \rightarrow a_{1}^{-}(1260) K^{+}$ with $a_{1}^{ \pm}(1260) \rightarrow \pi^{+} \pi^{ \pm} \pi^{ \pm}$[9]. We also search for a direct $C P$ violation by measuring the charge asymmetry $\mathcal{A}_{\text {ch }}$, defined as $\left(\Gamma^{-}-\Gamma^{+}\right) /\left(\Gamma^{-}+\Gamma^{+}\right)$, in the decay rates $\Gamma^{ \pm}$for a charged $B$ meson, or $\Gamma^{+}\left(B^{0} \rightarrow a_{1}^{-}(1260) K^{+}\right)$and its charge conjugate for a neutral $B$ meson.

The data were collected with the $B A B A R$ detector [10] at the PEP-II asymmetric energy $e^{+} e^{-}$collider [11]. An integrated luminosity of $347 \mathrm{fb}^{-1}$, corresponding to (382.9 \pm $4.2) \times 10^{6} B \bar{B}$ pairs, was recorded at the $Y(4 S)$ resonance (on-resonance, center-of-mass energy $\sqrt{s}=10.58 \mathrm{GeV}$ ). An additional $37 \mathrm{fb}^{-1}$, recorded about $40 \mathrm{MeV}$ below the $\mathrm{Y}(4 S)$ resonance (off-resonance), is used for continuum background studies.

Charged particles are detected and their momenta measured by a combination of a silicon vertex tracker, consisting of five layers of double-sided silicon detectors, and a 40-layer central drift chamber, both operating inside the 1.5-T magnetic field of a superconducting solenoid. The tracking system covers $92 \%$ of the solid angle in the centerof-mass frame.

Photons and electrons are detected with a $\mathrm{CsI}(\mathrm{Tl})$ electromagnetic calorimeter. Charged-particle identification
(PID) is provided by the average energy loss $(d E / d x)$ measured in the tracking devices and by an internally reflecting ring-imaging Cherenkov detector (DIRC) covering the central region. A $K / \pi$ separation of more than 4 standard deviations $(\sigma)$ is achieved for momenta below $3 \mathrm{GeV} / c$, decreasing to $2.5 \sigma$ at the highest momenta of the $B$ decay products.

Monte Carlo (MC) simulations of the signal decay modes, continuum, $B \bar{B}$ backgrounds, and detector response [12] are used to establish the event selection criteria. Exclusive MC signal events are simulated as $B \rightarrow$ $a_{1}(1260) K$ with $a_{1}(1260) \rightarrow \rho \pi$. For the $a_{1}(1260)$ meson parameters we take the mass $m_{0}=1230 \mathrm{MeV} / c^{2}$ and $\Gamma_{0}=400 \mathrm{MeV} / c^{2}[1,13]$. We account for the uncertainties of these resonance parameters in the determination of systematic uncertainties. The $a_{1}^{+}(1260) \rightarrow \pi^{-} \pi^{+} \pi^{+}$decay proceeds mainly through the intermediate states $(\pi \pi)_{\rho} \pi$ and $(\pi \pi)_{\sigma} \pi$ [14]. No attempt is made to separate the contributions of the dominant $P$-wave $(\pi \pi)_{\rho}$ from the $S$-wave $(\pi \pi)_{\sigma}$ in the channel $\pi^{+} \pi^{-}$. A systematic uncertainty is estimated due to the difference in the selection efficiency.

We reconstruct the decay $a_{1}^{+}(1260) \rightarrow \pi^{-} \pi^{+} \pi^{+}$with the following requirement on the invariant mass: $0.87<$ $m_{a_{1}}<1.5 \mathrm{GeV} / c^{2}$ for $B^{0} \rightarrow a_{1}^{-}(1260) K^{+}$and $0.87<$ $m_{a_{1}}<1.8 \mathrm{GeV} / c^{2}$ for $B^{+} \rightarrow a_{1}^{+}(1260) K^{0}$. The different $a_{1}$ mass selections are motivated by charm background studies. The intermediate $\pi^{+} \pi^{-}$state is reconstructed with an invariant mass between 0.51 and $1.1 \mathrm{GeV} / c^{2}$. Secondary $a_{1}(1260)$ daughter pions are rejected if their PID signatures satisfy requirements for being consistent with protons, electrons, or kaons. PID requirements ensure the identity of the primary charged kaon. Candidate $K_{S}^{0} \rightarrow$ $\pi^{+} \pi^{-}$decays are formed from pairs of oppositely charged tracks with $0.486<m_{\pi \pi}<0.510 \mathrm{GeV} / c^{2}$, having a decay vertex $\chi^{2}$ probability greater than 0.001 , and a reconstructed decay length larger than 3 times its uncertainty.

We reconstruct the $B$-meson candidate by combining an $a_{1}(1260)$ candidate and a charged or neutral kaon. A $B$-meson candidate is characterized kinematically by the energy-substituted mass $m_{\mathrm{ES}}=\sqrt{\left(s / 2+\mathbf{p}_{0} \cdot \mathbf{p}_{B}\right)^{2} / E_{0}^{2}-\mathbf{p}_{B}^{2}}$ and energy difference $\Delta E=E_{B}^{*}-\frac{1}{2} \sqrt{s}$, where the subscripts 0 and $B$ refer to the $Y(4 S)$ and the $B$ candidate in the laboratory frame, respectively, and the asterisk denotes the $Y(4 S)$ frame. The resolutions in $m_{\mathrm{ES}}$ and $\Delta E$ are about $3.0 \mathrm{MeV} / c^{2}$ and $20 \mathrm{MeV}$, respectively. We require $|\Delta E| \leq$ $0.1 \mathrm{GeV}$ and $5.25 \leq m_{\mathrm{ES}} \leq 5.29 \mathrm{GeV} / c^{2}$. To reduce fake $B$-meson candidates we require a $B$ vertex $\chi^{2}$ probability larger than 0.01 . The cosine of the angle between the direction of the $\pi$ meson from $a_{1}(1260) \rightarrow \rho \pi$ with respect to the flight direction of the $B$ in the $a_{1}(1260)$ meson rest frame is required to be between -0.85 and 0.85 to 
suppress combinatorial background. The distribution of this variable is flat for signal and peaks near \pm 1 for this background.

To reject continuum background, we use the angle $\theta_{T}$ between the thrust axis of the $B$ candidate and that of the rest of the tracks and neutral clusters in the event, calculated in the center-of-mass frame [15]. The distribution of $\cos \theta_{T}$ is sharply peaked near \pm 1 for combinations drawn from jetlike $q \bar{q}$ pairs and is nearly uniform for the isotropic $B$-meson decays; we require $\left|\cos \theta_{T}\right|<0.65$. The remaining continuum background is modeled from off-resonance data.

We use MC simulations of $B^{0} \bar{B}^{0}$ and $B^{+} B^{-}$decays to study $B \bar{B}$ backgrounds, which can come from both charmless and charmed decays. The modes $B^{0} \rightarrow a_{2}^{-}(1320) K^{+}$ and $B^{+} \rightarrow a_{2}^{+}(1320) K^{0}$ decay to the same final states as the signal modes. We suppress these backgrounds with the angular variable $\mathcal{H}$, defined as the cosine of the angle between the normal to the plane of the $3 \pi$ resonance and the flight direction of the $K$ meson evaluated in the $3 \pi$ resonance rest frame. Since the $a_{1}(1260)$ and $a_{2}(1320)$ have spins of 1 and 2, respectively, the distributions of the variable $\mathcal{H}$ for these two resonances differ. We require $|\mathcal{H}|<0.62$.

We have on average 1.3 candidates per event for both signal decay modes and we select the $B$ candidate with the highest $B$ vertex probability. From the MC simulation we find that the best candidate selection algorithm finds the correct-combination candidate in $92 \%$ of both signal decay modes and that it induces negligible bias.

We use unbinned, multivariate maximum-likelihood (ML) fits to measure the yields of $B^{+} \rightarrow a_{1}^{+}(1260) K^{0}$ and $B^{0} \rightarrow a_{1}^{-}(1260) K^{+}$. The likelihood function incorporates five variables. We describe the $B$ decay kinematics with the two above-mentioned variables $\Delta E$ and $m_{\mathrm{ES}}$, as well as the invariant mass of the $3 \pi$ system, a Fisher discriminant $\mathcal{F}$ and the variable $\mathcal{H}$. The Fisher discriminant combines four variables: the angles with respect to the beam axis in the $\Upsilon(4 S)$ frame of the $B$ momentum and $B$ thrust axes and the zeroth and second angular moments $L_{0,2}$ with respect to the thrust axis of the $B$ candidate [16]. Since the correlation between the observables in the selected data and in MC signal events is small, we take the probability density function (PDF) for each event to be a product of the PDFs for the individual observables. Corrections for the effects of possible correlations are made on the basis of MC studies described later. The selected data samples besides the signal events contain continuum $q \bar{q}$ and $B \bar{B}$ combinatorial background.

The $B \bar{B}$ background has the following components in the likelihood: charmless, charm, and $a_{2}^{-}(1320) K^{+}$. There are also three additional components: $f_{0} K, \rho^{0} K$ with their yields fixed to the value determined from the measured branching fractions [17], and the nonresonant $\rho^{0} \pi^{+} K$ with a yield fixed in the fit to the value expected using an assumed branching fraction of $(2.0 \pm 2.0) \times 10^{-6}$. We account for the uncertainties of these branching fractions in the determination of the systematic uncertainties. A charged particle from a signal event may be exchanged with a charged particle from the rest of the event. These socalled self-cross feed (SCF) events are considered background events. The charmless $B \bar{B}$ background has a dependence on the ML fit observables that is similar to that for SCF events, and thus the SCF events can be modeled as part of the charmless component.

The likelihood function is defined as

$$
\begin{aligned}
\mathcal{L}= & \exp \left(-\sum_{k} n_{k}\right) \prod_{i=1}^{N} \sum_{k} n_{k} \\
& \times \mathcal{P}_{k}\left(m_{\mathrm{ES}}^{i}\right) \mathcal{P}_{k}\left(\Delta E^{i}\right) \mathcal{P}_{k}\left(\mathcal{F}^{i}\right) \mathcal{P}_{k}\left(m_{a_{1}}^{i}\right) \mathcal{D}_{k}\left(\mathcal{H}^{i}\right),
\end{aligned}
$$

where $N$ is the total number of events in the fit sample, $n_{k}$ is the yield fitted for the likelihood component $k$, and $P_{k}\left(x^{i}\right)$ is the PDF for observable $x$ in event $i$. We determine the PDFs for signal and $B \bar{B}$ backgrounds from MC distributions in each observable. For the continuum background we establish the functional forms and initial parameter values of the PDFs with off-resonance data. The PDF of the invariant mass of the $a_{1}(1260)$ meson in signal events is parametrized as a relativistic Breit-Wigner line shape with a mass-dependent width which takes into account the effect of the mass-dependent $\rho$ width [18]. We fix the $a_{1}(1260)$ meson parameters to the values found in the branching fraction measurement of $B^{0} \rightarrow a_{1}^{ \pm} \pi^{\mp}$ [1]. The PDF of the invariant mass of the $a_{2}(1320)$ meson is parametrized by a relativistic Breit-Wigner distribution. The $m_{\mathrm{ES}}$ and $\Delta E$ distributions for signal are parametrized as double Gaussian functions. The $\Delta E$ distribution for continuum background is parametrized by a linear function. The $m_{\mathrm{ES}}$ distribution for the combinatorial background is described by an empirical function that accounts for threshold effects [19]. We model the Fisher distribution $\mathcal{F}$ using a Gaussian function with different widths above and below the mean. The $\mathcal{H}$ distributions are modeled using polynomials.

In the fit for the decay $B^{0} \rightarrow a_{1}^{-}(1260) K^{+}\left[B^{+} \rightarrow\right.$ $\left.a_{1}^{+}(1260) K^{0}\right]$ there are, respectively, fourteen (twelve) free parameters: five (five) yields and nine (seven) parameters affecting the shape of the combinatorial background. Table I lists the results of the fits. We measure the signal yield bias by generating and fitting $\mathrm{MC}$ simulated samples containing signal and background populations expected from data. The signal reconstruction efficiency is obtained from the fraction of correctly reconstructed signal MC events passing the selection criteria. Branching fractions for each decay are computed by subtracting the fit bias from the measured yield, and dividing the result by the efficiency, the daughter branching fraction product, and the number of $B \bar{B}$ pairs produced. Equal production rates to $B^{0} \bar{B}^{0}$ and $B^{+} B^{-}$pairs are assumed. The significance is 
TABLE I. Number of events $N$ in the sample, fitted signal yield, and measured bias (to be subtracted from the signal yield) in events (ev.), detection efficiency $(\epsilon)$, daughter branching fraction product $\prod \mathcal{B}_{i}$, significance $(S)$ (systematic uncertainties included), the products of the branching fractions $\mathcal{B}\left(B^{0} \rightarrow a_{1}^{-}(1260) K^{+}\right) \mathcal{B}\left(a_{1}^{-}(1260) \rightarrow \pi^{+} \pi^{-} \pi^{-}\right)$and $\mathcal{B}\left(B^{+} \rightarrow\right.$ $\left.a_{1}^{+}(1260) K^{0}\right) \mathcal{B}\left(a_{1}^{+}(1260) \rightarrow \pi^{-} \pi^{+} \pi^{+}\right)$, respectively, and charge asymmetry with statistical and systematic error.

\begin{tabular}{lcc}
\hline \hline \multicolumn{1}{c}{ Parameter } & $a_{1}^{-}(1260) K^{+}$ & $a_{1}^{+}(1260) K^{0}$ \\
\hline$N$ (ev.) & 12196 & 9468 \\
Signal yield (ev.) & $272 \pm 44$ & $241 \pm 32$ \\
Bias (ev.) & +24 & +18 \\
$\epsilon(\%)$ & 7.9 & 9.6 \\
$\prod \mathcal{B}_{i}(\%)$ & 100.0 & 34.6 \\
$S(\sigma)$ & 5.1 & 6.2 \\
$\mathcal{B}\left(\times 10^{-6}\right)$ & $8.2 \pm 1.5 \pm 1.2$ & $17.4 \pm 2.5 \pm 2.2$ \\
$\mathcal{A}_{\mathrm{ch}}$ & $-0.16 \pm 0.12 \pm 0.01$ & $0.12 \pm 0.11 \pm 0.02$ \\
\hline \hline
\end{tabular}

taken as the square root of the difference between the value of $-2 \ln \mathcal{L}$ (with systematic uncertainties included) for zero signal and the value at its minimum.

In Figs. 1 and 2 we show the $\Delta E, m_{\mathrm{ES}}, m_{a_{1}}$, and $\mathcal{F}$ projections made by selecting events with a signal likelihood (computed without the variable shown in the figure) exceeding a threshold that optimizes the expected sensitivity.

Most of the systematic errors on the signal yield arising from uncertainties in the values of the PDF parameters are already incorporated into the overall statistical error, since they are floated in the fit. The systematic error on the $B^{0} \rightarrow$ $a_{1}^{-}(1260) K^{+} \quad\left[B^{+} \rightarrow a_{1}^{+}(1260) K^{0}\right]$ fit yield is 28 (23) events, which is obtained by varying the PDF parameters within their uncertainties. We estimate the uncertainty arising from the number of $B \bar{B}$ pairs to be $1.1 \%$. The
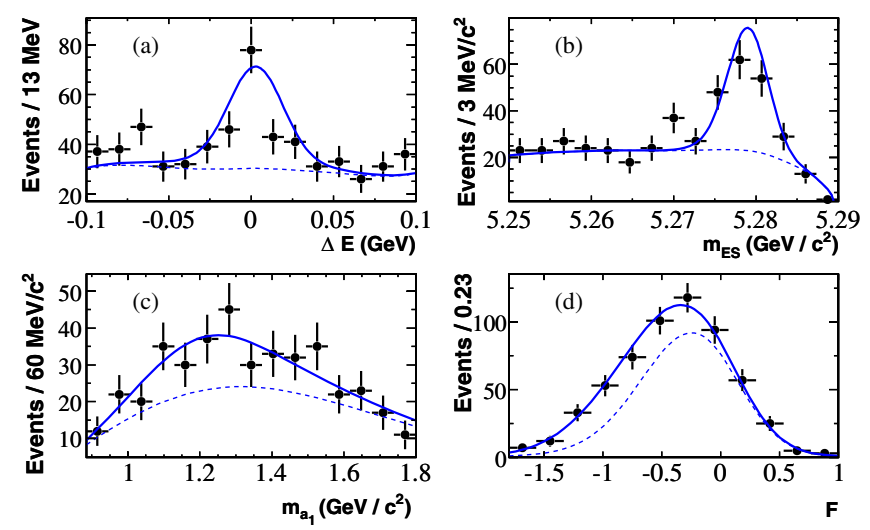

FIG. 1 (color online). Projections of (a) $\Delta E$, (b) $m_{\mathrm{ES}}$, (c) $m_{a_{1}}$, and (d) $\mathcal{F}$ for the $B^{+} \rightarrow a_{1}^{+}(1260) K^{0}$ decay mode. Points represent on-resonance data, dashed lines the continuum and $B \bar{B}$ backgrounds, and solid lines the full fit function. These plots are made with a cut on the signal likelihood which includes $35 \%-45 \%$ of the signal. uncertainty in the fit bias correction is 12 (9) events, taken as half of the fit bias correction. The uncertainty in the $a_{1}(1260)$ meson parameters is 12 (6) events. The systematic uncertainty assigned to the fixed yields in the fit is 3 (4) events. The systematic effect due to differences between data and $\mathrm{MC}$ simulations for the $\cos \theta_{\mathrm{T}}$ selection is $1.8 \%$. A systematic uncertainty of $2.0(2.5) \%$ is evaluated for the PID. The tracking efficiency contributes to the systematics with $1.8(1.3) \%$. A systematic uncertainty of $2.5 \%$ is estimated for the difference in selection efficiency in the decay modes through the dominant $P$-wave $(\pi \pi)_{\rho}$ and the $S$-wave $(\pi \pi)_{\sigma}$. The contribution of interference between $a_{2}(1320)$ and $a_{1}(1260)$ is negligible. In fact, varying the $a_{2}(1320) K$ background with different selection criteria on the angular variable $\mathcal{H}$ gives no significant change to the efficiency-corrected signal yield of $a_{1}(1260) K$. We find also that the systematic effect due to different form factors in MC signal simulation is negligible. The total systematic error on the branching fraction of the charged (neutral) mode is $14 \%(13 \%)$.

The primary sources of systematic uncertainties in the charge asymmetry measurement are the track reconstruction or particle identification, the imperfect modelling of the interactions with material in the detector and the $B \bar{B}$ background. We study these systematic uncertainties with MC signal events, $q \bar{q}$ background in the data, and control samples. We assign a systematic uncertainty of $0.02(0.01)$ to the charge asymmetry of the charged (neutral) mode.

In summary, we have measured the branching fractions $\mathcal{B}\left(B^{+} \rightarrow a_{1}^{+}(1260) K^{0}\right) \mathcal{B}\left(a_{1}^{+}(1260) \rightarrow \pi^{-} \pi^{+} \pi^{+}\right)=$ $(17.4 \pm 2.5 \pm 2.2) \times 10^{-6}$ and $\mathcal{B}\left(B^{0} \rightarrow a_{1}^{-}(1260) K^{+}\right) \times$ $\mathcal{B}\left(a_{1}^{-}(1260) \rightarrow \pi^{+} \pi^{-} \pi^{-}\right)=(8.2 \pm 1.5 \pm 1.2) \times 10^{-6}$. The charged (neutral) $B$ decay mode is observed with a significance of 6.2 (5.1) standard deviations, which includes systematic uncertainties. We find no evidence for
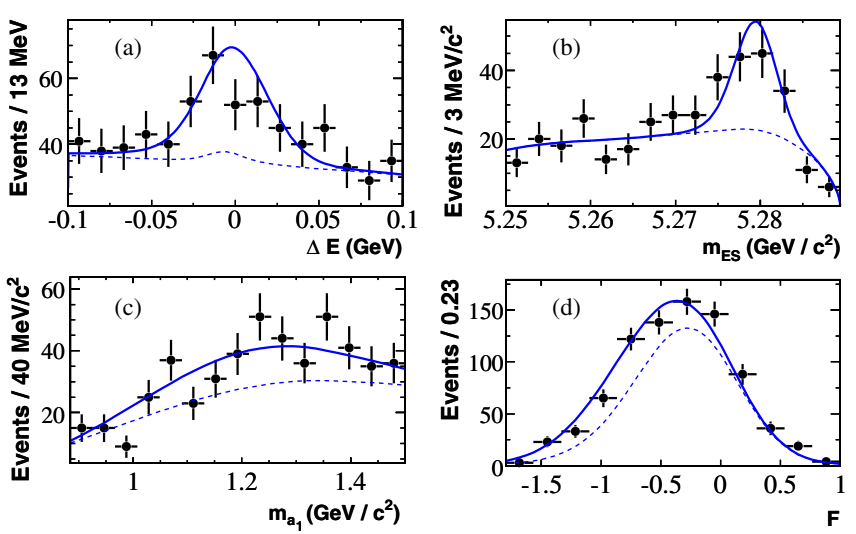

FIG. 2 (color online). Projections of (a) $\Delta E$, (b) $m_{\mathrm{ES}}$, (c) $m_{a_{1}}$, and (d) $\mathcal{F}$ for the $B^{0} \rightarrow a_{1}^{-}(1260) K^{+}$decay mode. Points represent data, dashed lines the continuum and $B \bar{B}$ backgrounds, and solid lines the full fit function. These plots are made with a cut on the signal likelihood which includes $25 \%-35 \%$ of the signal. 
a direct $C P$-violating asymmetry in these decay modes. Assuming $\mathcal{B}\left(a_{1}^{ \pm}(1260) \rightarrow \pi^{\mp} \pi^{ \pm} \pi^{ \pm}\right) \quad$ is equal to $\mathcal{B}\left(a_{1}^{ \pm}(1260) \rightarrow \pi^{ \pm} \pi^{0} \pi^{0}\right), \quad$ and $\quad$ that $\quad \mathcal{B}\left(a_{1}^{ \pm}(1260) \rightarrow\right.$ $\left.(3 \pi)^{ \pm}\right)$is equal to $100 \%$ [14], we obtain $\mathcal{B}\left(B^{0} \rightarrow\right.$ $\left.a_{1}^{-}(1260) K^{+}\right)=(16.3 \pm 2.9 \pm 2.3) \times 10^{-6}$ and $\mathcal{B}\left(B^{+} \rightarrow\right.$ $\left.a_{1}^{+}(1260) K^{0}\right)=(34.9 \pm 5.0 \pm 4.4) \times 10^{-6}$. These results are in reasonable agreement with the theoretical estimates.

We are grateful for the excellent luminosity and machine conditions provided by our PEP-II colleagues, and for the substantial dedicated effort from the computing organizations that support BABAR. The collaborating institutions wish to thank SLAC for its support and kind hospitality. This work is supported by DOE and NSF (U.S.A.), NSERC (Canada), IHEP (China), CEA and CNRS-IN2P3 (France), BMBF and DFG (Germany), INFN (Italy), FOM (The Netherlands), NFR (Norway), MIST (Russia), and STFC (U.K.). Individuals have received support from CONACyT (Mexico), Marie Curie EIF (European Union), the A.P. Sloan Foundation, the Research Corporation, and the Alexander von Humboldt Foundation.

\footnotetext{
*Deceased

†Present address: Tel Aviv University, Tel Aviv, 69978, Israel.

${ }^{\ddagger}$ Also at Università di Perugia, Dipartimento di Fisica, Perugia, Italy.

${ }^{\S}$ Also at Università della Basilicata, Potenza, Italy.

"Also at Universitat de Barcelona, Facultat de Fisica, Departament ECM, E-08028 Barcelona, Spain.

[1] B. Aubert et al. (BABAR Collaboration), Phys. Rev. Lett. 97, 051802 (2006).

[2] B. Aubert et al. (BABAR Collaboration), Phys. Rev. Lett. 98, 181803 (2007).

[3] N. Cabibbo, Phys. Rev. Lett. 10, 531 (1963); M. Kobayashi and T. Maskawa, Prog. Theor. Phys. 49, 652 (1973).
}

[4] J. Charles et al. (CKMfitter Group), Eur. Phys. J. C 41, 1 (2005).

[5] M. Gronau and J. Zupan, Phys. Rev. D 73, 057502 (2006).

[6] H.-Y. Cheng and K.-C. Yang, Phys. Rev. D 76, 114020 (2007).

[7] V. Laporta, G. Nardulli, and T. N. Pham, Phys. Rev. D 74, 054035 (2006); 76, 079903(E) (2007).

[8] For the decay $B^{+} \rightarrow a_{1}^{+}(1260) K^{0}$ the mentioned estimated range of branching fractions is obtained using the experimental measurement of the branching fraction of the channel $B^{+} \rightarrow \pi^{+} K^{0}$ assuming naive factorization. See Ref. [7] for more details.

[9] Charge-conjugate reactions are implied throughout this paper.

[10] B. Aubert et al. (BABAR Collaboration), Nucl. Instrum. Methods Phys. Res., Sect. A 479, 1 (2002).

[11] PEP-II Conceptual Design Report, SLAC Report No. SLAC-R-418, 1993.

[12] BABAR detector MC simulation is based on GEANT4: S. Agostinelli et al., Nucl. Instrum. Methods Phys. Res., Sect. A 506, 250 (2003).

[13] EvtGen particle decay simulation package, D. J. Lange, Nucl. Instrum. Methods Phys. Res., Sect. A 462, 152 (2001).

[14] Y.-M. Yao et al. (Particle Data Group), J. Phys. G 33, 1 (2006).

[15] S. Brandt et al., Phys. Lett. 12, 57 (1964); E. Fahri, Phys. Rev. Lett. 39, 1587 (1977).

[16] B. Aubert et al. (BABAR Collaboration), Phys. Rev. D 70, 032006 (2004).

[17] B. Aubert et al. (BABAR Collaboration), Phys. Rev. Lett. 97, 201801 (2006).

[18] T. A. Armstrong et al. (WA76 Collaboration), Z. Phys. C 48, 213 (1990).

[19] With $x \equiv m_{\mathrm{ES}} / E_{b}$ and $\xi$ a parameter to be fitted, $f(x) \propto$ $x \sqrt{1-x^{2}} \exp \left[-\xi\left(1-x^{2}\right)\right]$. See H. Albrecht et al. (ARGUS Collaboration), Phys. Lett. B 241, 278 (1990). 